\title{
Mass or molar? Recommendations for reporting concentrations of therapeutic drugs
}

Graham R D Jones MB BS, DPhil, FRCPA, Chemical Pathologist ${ }^{1}$

Stewart Bryant MB BS, FRCPA, FAMM, Transfusion Medicine Specialist ${ }^{2}$

Robert Fullinfaw MSc,
Senior Scientist
3

Ken llett BPharm, PhD Emeritus Professor of Pharmacology ${ }^{4}$

John O Miners

PhD, DSc, Professor and Head of Department of Clinical Pharmacology ${ }^{5}$

Raymond G Morris $\mathrm{PhD}, \mathrm{FFSC}(\mathrm{RCPA})$ Affiliate Professor, Clinical Pharmacology ${ }^{6}$

\section{Matthew P Doogue MBChB, FRACP, Clinical Pharmacologist and Endocrinologist ${ }^{5}$ \\ 1 SydPath, St Vincent's Hospital, Sydney, NSW. \\ 2 Australian Red Cross Blood Service, Brisbane, QLD. \\ 3 Royal Melbourne Hospital, \\ Melbourne, VIC \\ 4 University of Western Australia, Perth, WA. \\ 5 Flinders University, Adelaide, SA. \\ 6 University of Adelaide, Adelaide, SA. \\ gjones@ \\ stvincents.com.au}

doi: 10.5694/mjal2.10366 nits used to report any measurement are an integral component of the result. Errors in communication of units have caused catastrophic failures, for example the Mars Climate Orbiter spacecraft crash in 1999. ${ }^{1}$ One area of laboratory medicine where different units are in common use is therapeutic drug concentrations, where results for the same drug may be reported in either mass units (eg, mg/L) or molar units (eg, $\mu \mathrm{mol} / \mathrm{L})$ by different laboratories, (see Box for a note on the prefix micro $[\mu])$.

Clinical errors may occur if a result in one unit is interpreted using information expressed in a different unit. This situation may occur when a result is separated from its report (for example, telephoned results), or when a clinician is unaware of unit differences when consulting a textbook, journal article, guideline or website. When assessing results in the toxic range it is more likely that external references will be used, as the therapeutic intervals provided on laboratory reports generally do not indicate the severity of possible toxicity. With the rise of health databases where results from more than one laboratory may be combined, uniformity of reporting takes on even greater importance.

A recent publication highlighting variation in units used for reporting drug concentrations, ${ }^{2}$ as well as reports of patient harm due to misinterpretation of drug concentrations, led to the establishment of a working party (WP) to address this issue.

\section{Recommendation development process}

A WP was established by the Royal College of Pathologists of Australasia (RCPA), the Australasian Association of Clinical Biochemists (AACB), the Australasian Society of Clinical and Experimental Pharmacologists and Toxicologists (ASCEPT) and the Royal Australasian College of Physicians. The goal of the WP was to recommend uniform units for reporting drug concentration measurements by pathology laboratories in Australia and New Zealand.

The process adopted by the WP was to systematically identify and evaluate all relevant issues. In assessing information, the WP considered patient safety and clinical decision making to be the most important issues. A discussion paper was developed for consultation and distributed to the parent societies and other interested parties. Over 20 written responses and several verbal responses were received. In light of these responses, the document was revised using a consensus approach. Dur-
Summary

A working party (WP) from the Australasian Association of Clinical Biochemists, Australasian Society of Clinical and Experimental Pharmacologists and Toxicologists, Royal College of Pathologists of Australasia and Royal Australasian College of Physicians recommends the following:

- mass units should be used for reporting therapeutic drug concentrations in Australia and New Zealand; and

- the litre $(L)$ should be used as the denominator when expressing concentration. Examples of these units are $\mathrm{mg} / \mathrm{L}$ and $\mu \mathrm{g} / \mathrm{L}$.

Exceptions to these principles include:

- drugs for which there is current uniformity of reporting and supporting information using molar units, notably lithium ( $\mathrm{mmol} / \mathrm{L})$ and methotrexate $(\mu \mathrm{mol} / \mathrm{L})$;

- drugs that are also present as endogenous substances, where the units used routinely should continue to be used. This applies to many substances, including minerals (eg, iron; $\mu \mathrm{mol} / \mathrm{L}$ ), vitamins (eg, vitamin D; nmol/L) and hormones (eg, thyroxine; pmol/L).

- drugs for which the denominator is not a volume of fluid and there is international uniformity of reporting (eg, thiopurine metabolites; per $10^{9}$ red blood cells).

These recommendations relate to drugs that are used therapeutically, whether measured for therapeutic drug monitoring purposes or for assessment of overdose. Other substances, such as drugs of misuse, heavy metals or environmental toxins, were not considered by the WP and are thus not covered by this document.

These recommendations should also be applied to other supporting documentation such as published guidelines, journal articles and websites. The implementation of these recommendations in New Zealand is subject to local confirmation.

ing this period, presentations were also made at the AACB Annual Scientific Conference, the RCPA annual meeting and a combined societies therapeutic drug monitoring workshop. The completed document was then formally endorsed by the four parent bodies represented on the $\mathrm{WP}^{3}$

\section{Considerations in recommendations}

\section{Australian and international recommendations}

In 1986 an RCPA broadsheet on the introduction of International System of Units (SI) reporting recommended the use of molar units for reporting therapeutic drug concen- 
trations. ${ }^{4}$ ASCEPT recommends the use of mass units; however, this recommendation has not been published. The Australian National Measurement Institute (NMI) is responsible for the implementation of SI reporting of units in Australia; however, it offers no specific guidance on the issue of drug measurements in serum. Both mass (kilograms) and amount (moles) are fundamental quantities in the SI system. Standards Australia has no documents related to serum drug concentrations; however, the Australian standard for urine toxicology (AS4308) specifies cut-offs in mass units. ${ }^{5}$

The issue of units for drug measurements was recently considered at a consensus meeting in the United Kingdom, where it was recommended that mass units be used for routine reporting, with the litre (L) used as the denominator, for all drugs, whether for therapeutic or toxicological purposes. ${ }^{6}$ Specific exceptions were made for thyroxine, lithium, methotrexate and iron, where molar units were recommended. The International Federation of Clinical Chemistry and Laboratory Medicine (IFCC) and International Union of Pure and Applied Chemistry (IUPAC) specify molar units as the agreed communication terminology for therapeutic drugs. ${ }^{7}$ The purpose of their standard is not specifically to guide reporting of patient results for clinical use, but to provide unambiguous terminology for data exchange.

There are several important international organisations in the field of medicine regulation that do not have formal recommendations on this topic but generally use mass units in publications covering pharmacokinetic or similar studies. These include the Food and Drug Administration in the United States ${ }^{8}$ and the International Conference on Harmonisation of Technical Requirements for Registration of Pharmaceuticals for Human Use. ${ }^{9}$ Although not specifically related to therapeutic use of drugs, the World AntiDoping Agency specifies the use of mass units in its relevant documentation. ${ }^{10,11}$

\section{Units used in published literature, textbooks and other reference sources}

In clinical practice, drug measurement results are compared with information from a range of reference sources including journals, textbooks, websites, guidelines and handbooks. These may have been produced locally for use in Australia and New Zealand or sourced from overseas.

A review by the WP of the units used in major journals and textbooks found a wide variation in practice, with a tendency for those produced in the US to use mass units, and for references from other locations, including Australia, to use a combination of mass and molar units, often without internal consistency. ${ }^{3}$ This variation is not unexpected given the recommendation from the International Committee of Medical Journal Editors, which states: "Drug concentrations may be reported in either mass or SI units, but the alternative should be provided in parentheses where appropriate." ${ }^{\prime 2}$ It is now common for international textbooks to provide both units for some drugs, at least in a summary table.

The most widely available first-line references for drug information in Australia are the Australian medicines handbook, ${ }^{13}$ MIMS (http://www.mims. com.au) and the Therapeutic Guidelines series (http://www.tg.org.au/). These works use a mixture of molar and mass units and often both. ${ }^{2}$ The Therapeutic Goods Administration approves a product information (PI) document for all drugs available in Australia (available through www.ebs.tga.gov.au), which is presented through MIMS and in common software packages for general practitioners, such as Best Practice (Best Practice Software) or Medical Director (Health Communication Network). Another review body, Phoenix Medical Publishing, prepares summaries that are available online through the AusDI online reference tool. The PI and AusDI sections on pharmacokinetics, dosing and toxicity most commonly use mass units, although molar units are also provided for some common drugs.

\section{Current reporting practice}

A review of practice in Australia has shown that the concentrations of some drugs are almost always reported in mass units (eg, gentamicin), while those of other drugs are always reported in molar units (eg, lithium and methotrexate). However, for some drugs, both systems are used to a significant extent (eg, phenytoin and paraceta$\mathrm{mol}$ ). Our personal experiences suggest that for some classes of drugs not included in the published study, ${ }^{3}$ such as immunosuppressants, concentrations are reported almost entirely in mass units, as are those of many specialised drugs measured in dedicated clinical pharmacology laboratories.

In areas outside the routine pathology environment, for example forensic pathology, drug concentrations are routinely reported in mass units.

With regard to international reporting practice, members of the WP sought information from international colleagues on practice in their countries. In the US, mass units are used almost universally, although $\mathrm{mL}$ and $\mathrm{dL}$ are frequently used as the denominator. In some countries, for example Sweden and Canada, there is a similar situation to that found in New Zealand, with molar units used for a limited number of drugs and mass units used for the remainder. The UK is becoming a consistent user of mass units, with specified exceptions, as their agreed guidelines are implemented. ${ }^{14}$ The WP did not identify any locations with full or planned use of molar units for all drugs.

\section{Relationship with units used for prescribing drugs}

Mass units are almost universally used for describing drug dosages. There are exceptions, such as vitamin D, heparin and insulin, which are prescribed in international units. The dosing unit is relevant to drug reporting when there is a direct relationship between the numerical value of the result and the dose or change in dose prescribed.

In or near the therapeutic range, drug concentrations usually vary in direct proportion to dose and therefore drug dose can be considered relative to the therapeutic range. For example, a drug present at twice the target concentration can be adjusted by halving the dose. The units used for the concentration and the dosing do not need to be aligned for this type of dose adjustment.

However, there are a number of exceptions to this that can cause both within-patient and between-patient variability in drug concentrations:

- drug factors such as saturable clearance (eg, phenytoin) or saturable protein binding (eg, valproate); 
- patient factors such as hypoalbuminaemia (due to liver dysfunction or nephrotic syndrome) or competition with binding sites (due to uraemia or coprescribed drugs); and

- higher drug concentrations such as those occurring in overdose.

In these cases the drug concentration may not be directly proportional to drug dose, requiring a more complex application of pharmacokinetics to interpret drug concentrations.

At steady state, drug concentrations are directly related to dose via drug clearance. Physicians and pharmacists are expected to understand this pharmacokinetic relationship, which for parenteral dosing can be described as follows:

- maintenance dose $=$ systemic clearance $\times$ target con centration at steady state;

- similarly, drug concentration after a loading dose is determined by apparent volume of distribution; and

- loading dose = volume of distribution $\times$ target concentration.

Thus the dose and target concentration are linked by a constant for which reference values can be obtained. When performing calculations it is feasible to convert between units, but the process is simplified when common units are used. Note that the unit of volume used for pharmacological constants such as volume of distribution is the litre, supporting the case for litre to be the volume unit for drug concentrations.

Drugs, prodrugs, metabolites and binding proteins interact on the basis of moles rather than mass. Therefore, it may be expected that reporting in molar concentrations may assist with understanding the relative concentrations of these components. However, as there is variable bioactivity of different metabolites, and the concentration of serum albumin, the most common drug ligand, is reported in mass units, there are significant limitations to the benefits of reporting in molar units for this purpose.

\section{Assay calibration}

In order to produce universally comparable results, laboratory assays must use calibrators that are traceable to international reference materials or methods, such as those listed on the database of the Joint Committee for Traceability in Laboratory Medicine, ${ }^{15}$ or obtained from sources such as the United States Pharmacopeia. ${ }^{16}$ IFCC and IUPAC guidelines ${ }^{7}$ and other sources ${ }^{17}$ provide molecular weights for many drugs for the purposes of conversion between mass and molar units. A limited number of drugs (eg, gentamicin) are mixtures of compounds, and their molecular weight is thus uncertain and may be dependent on the relative abundance of their various components, making assignment of the molar concentrations less reliable.

Some drugs are available in a number of different molecular complexes (eg, hydrates and varying salts). In these cases, when reporting in mass units, it is necessary to define the entity used to standardise the assay. Ideally this should be in the same form as that used to describe the dose of the drug, but it is common laboratory practice to calibrate against a standard based on the free base of the drug. This definition has not been universally adopted for drug dosing, for example phenytoin is referred to as both phenytoin and phenytoin sodium, with the molecular weights of 252 and 274, respectively. Guidance on exact definition of a drug is available from the World Health Organization through the International Pharmacopoeia. ${ }^{18}$

The use of molar units would reduce the potential for error in assay standardisation by eliminating uncertainty about the molecular form to be used. As assay calibration is generally carried out by trained personnel away from the urgency of acute clinical decision making, this is considered as having lower potential for error. However, there is a need for laboratories and authors to specify the molecular form of any drug used to standardise an assay or prepare a therapeutic dose.

\section{Other factors}

Some substances used therapeutically are also endogenous compounds that are measured for purposes other than therapeutic monitoring and toxicology. Examples include the hormones cortisol (nmol/L), testosterone (nmol/L) and thyroxine $(\mathrm{pmol} / \mathrm{L})$, the minerals iron $(\mu \mathrm{mol} / \mathrm{L})$ and calcium $(\mathrm{mmol} / \mathrm{L})$, and the vitamins $\mathrm{B}_{12}(\mathrm{pmol} / \mathrm{L})$ and $\mathrm{D}(\mathrm{nmol} / \mathrm{L})$. For these and other endogenous compounds, the units used for reporting these analytes should be the same regardless of endogenous or exogenous origin.

\section{Conclusions and overall recommendations}

The WP has concluded that it is important that serum concentrations for any drug are reported with one unit throughout Australia and New Zealand irrespective of the location of the laboratory or the purpose of the measurement. The aim is to avoid patient harm that may occur if a result is interpreted against a decision point reported in different units. We recommend the use of mass units for reporting drug concentrations, except where there are specific reasons for the use of other units for a specific therapeutic agent.

Although the only published Australasian recommendation, which dates from 1986, recommended molar units, the recommendation was followed by the statement that "It is rational and possibly a long term expectation that drugs also will be labeled and administered in molar terms". ${ }^{3}$ As drug dosing remains in mass units, without movement for change, it is appropriate to review this recommendation. The UK consensus is particularly relevant to the current situation as it relates directly to pathology reporting, is the product of wide consultation, is in a similar clinical context and has been produced recently.

Supporting information in the form of textbooks, journal articles, websites and guidelines tends to follow the common usage in the country of origin. As the US and UK are the major English language sources of information, it is likely that the predominance of mass units in these sources will become further entrenched. The officially approved PI used in Australia predominantly uses mass units, although some documents also use molar units in selected sections.

In circumstances where calculations are used to predict dose based on serum concentration, using the same units for both components removes the risk due to conversion between units. 
There are drugs for which units other than mass units are preferred because of universal usage of these units in laboratories and supporting information. These are:

- lithium (mmol/L);

- methotrexate $(\mu \mathrm{mol} / \mathrm{L})$;

- thiopurine metabolites (pmol/109 red blood cells); and

- drugs for which molar units are uniformly used locally and internationally in laboratories and supporting information.

For all drugs that are also present endogenously (including hormones, minerals and vitamins used therapeutically), we recommend the same unit as is used for routine clinical measurement.

We recommend that the litre should be the denominator for reporting drug concentrations unless there are specific reasons for using other units. In summary, the reasons for this recommendation are as follows. The litre is the SI unit for volume, is used for most other laboratory concentration measurements, and is commonly used for physiological volumes such as extracellular fluid volume and for volume of distribution.

The recommendations presented here are consistent with a wider project by the RCPA to standardise units of reporting for all pathology tests in Australia. ${ }^{19}$

\section{Use of the prefix micro $(\mu)$}

The Royal College of Pathologists of Australasia has recently adopted as policy the use of $\mathrm{u}$, rather than the Greek letter $\mu$, for the expression of "micro" in units for reporting pathology results (eg, ug/L rather than $\mu g / L$ for micrograms per litre). This is to ensure the safe transmission of the unit to different computer systems and printers, including those with a limited character set, and also to ensure consistency with the adoption of the Unified Code for Units of Measure (UCUM) system for the unambiguous electronic transmission of units.

Competing interests: Graham Jones has received research support from Roche Diagnostics, and travel support from Abbott Diagnostics, Roche Diagnostics and Bio-Rad.

Provenance: Not commissioned; externally peer reviewed.

1 Lloyd R. Metric mishap caused loss of NASA orbiter. CNN.com [internet] 1999. 30 Sep. http://edition.cnn.com/TECH/space/9909/30/mars.metric.02/ (accessed Sep 2011).

2 Jones GR. Reporting units for therapeutic drug monitoring: a correctable source of potential clinical error. Med J Aust 2007; 186: 420-421.

3 Bryant S, Doogue M, Fullinfaw R, et al. Reporting drug concentrations: mass or molar units? A recommendation from the AACB, ASCEPT, RCPA, RACP Common Units for Reporting Drug Concentrations Working Party. 2010.
http://www.sydpath.stvincents.com.au/MassvMolar-

ProposalForAdoption.pdf (accessed Nov 2012).

4 Royal College of Pathologists of Australasia. SI units revisited. Broadsheet No. 29. Sydney: RCPA, 1986.

5 Standards Australia and Standards New Zealand. Procedures for specimen collection and the detection and quantitation of drugs of abuse in urine. AS/NZS 4308: 2008.

6 Watson I, Barth J. Consensus meeting on units for reporting drug concentrations. ACB News 2006; 522: 14-15.

7 International Federation of Clinical Chemistry and Laboratory Medicine and International Union of Pure and Applied Chemistry. Properties and units in the clinical laboratory sciences. Part XII: Properties and units in clinical pharmacology and toxicology. Pure Appl Chem 2000; 72: 479-552. http:// media.iupac.org/publications/labinfo/English/Documents/7203olesen 479.pdf (accessed Sep 2011).

8 Food and Drug Administration. Formatting of bioequivalence summary tables. http://www.fda.gov/downloads/Drugs/DevelopmentApprovalProcess/ HowDrugsareDevelopedandApproved/ApprovalApplications/Abbreviated NewDrugApplicationANDAGenerics/UCM120957.pdf (accessed Sep 2011).

9 International Conference on Harmonisation of Technical Requirements for Registration of Pharmaceuticals for Human Use. Data elements and standards for drug dictionaries M5. http://www.ich.org/products/guidelines/ multidisciplinary/multidisciplinary-single/article/data-elements-andstandards-for-drug-dictionaries.html (accessed Sep 2011).

10 World Anti-Doping Agency Laboratory Committee. Minimum required performance levels for detection of prohibited substances. WADA technical document TD2010MRPL. 2010. http://www.wada-ama.org/documents/ world_anti-doping_program/wadp-is-laboratories/wada_td2010mrplv1.0 minimum\%20required\%20performance $\% 20$ levels_sept $\% 2001 \% 202010_{-}$ en.pdf (accessed Sep 2011).

11 World Anti-Doping Agency Laboratory Committee. Decision limits for the confirmatory quantification of threshold substances. WADA technical document TD2010DL. http://www.wada-ama.org/Documents/World_AntiDoping_Program/WADP-IS-Laboratories/Technical_Documents/WADATD2010DL-1.0-Decision-Limits-for-Confirmatory-Quantification-ofThreshold-Substances-EN.pdf (accessed Sep 2011).

12 International Committee of Medical Journal Editors. Uniform requirements for manuscripts submitted to biomedical journals: writing and editing for biomedical publication. Updated April 2010. http://www.icmje.org/urm_ full.pdf (accessed Sep 2011).

13 Rossi S, editor. Australian medicines handbook 2013. Adelaide: Australian Medicines Handbook Pty Ltd, 2013.

14 Barth J, Rae JK, Freedman D. Harmonisation of reference intervals. 2011. http://www.acb.org.uk/docs/Pathology\%20Harmony\%20for\%20web.pdf (accessed Sep 2011).

15 Joint Committee for Traceability in Laboratory Medicine. JCTLM database: laboratory medicine and in vitro diagnostics. http://www.bipm.org/jctlm/ (accessed Feb 2012).

16 United States Pharmacopeial Convention. USP reference standards listings \& catalog. http://www.usp.org/referenceStandards/catalog.html (accessed Sep 2011).

17 World Health Organization International Programme on Chemical Safety. Basic analytical toxicology. http://www.who.int/ipcs/publications/training poisons/basic_analytical_tox/en/indexl3.html (accessed Feb 2012).

18 The International Pharmacopoeia. 4th ed. 2011. http://apps.who.int/phint/ en/p/about/ (accessed Jul 2012)

19 Legg M, Swanepoel C. The Australian pathology units and terminology standardisation project - an overview. Clin Biochem Rev 2012; 33: 103-108. 口 